\title{
Endomarketing na Melhoria de Processos de Software Trabalho Técnico
}

\author{
Diogo Pinheiro de Araújo ${ }^{1}$, Josiane Brietzke Porto ${ }^{1}$ \\ ${ }^{1}$ Universidade do Vale do Rio dos Sinos (UNISINOS) \\ Av. Unisinos, 950 - 93022-000 - São Leopoldo - RS - Brasil \\ \{diogo_pinheiro13, josiane_brietzke\}@hotmail.com
}

\begin{abstract}
The competition between the software development enterprises is increasing and the organizations are looking for the qualifying of their processes, suiting their software development process to reference models, such as CMMI and MPS. However, there are aspects that should be taken under consideration, such as human factors, that can influence on results when trying to implement the processes. This paper analyzes the presence and importance of the instruments of Endomarketing in software improvement process programs, according to experts in the field, through a survey. The results of this research show us that the presence of the Endomarketing in software improvement process initiatives is lower than its importance.
\end{abstract}

Resumo. A concorrência austera entre empresas desenvolvedoras de software ocasiona a busca das mesmas por qualificação e adequação de seus processos às boas práticas de modelos como o MPS e o CMMI. Porém, existem aspectos que devem ser levados em consideração, como o fator humano, que podem influenciar projetos e iniciativas nessa área. Buscando tratar a influência do fator humano, esse trabalho analisou os principais instrumentos de Endomarketing presentes e considerados importantes na melhoria de processos de software, segundo a percepção de profissionais da área, em nível nacional, através de uma survey. Os resultados obtidos mostraram que a presença desses instrumentos na melhoria de processos de software está aquém do quão importante é considerado pelos especialistas.

\section{Introdução}

A melhoria de processos de software engloba o entendimento dos processos atuais de desenvolvimento de uma organização, identificando oportunidades de melhoria para reduzir custos, tempo e aumentar a qualidade do produto final [Sommerville 2011]. Ocorre que em iniciativas ou projetos nessa área, as organizações enfrentam a resistência por parte dos colaboradores, conforme observado em estudo anterior [Montoni e Rocha 2011].

Visto que o comprometimento dos colaboradores é essencial para o sucesso de uma iniciativa ou projeto nessa área e por outro lado existem poucas pesquisas sobre formas de amenizar ou tratar os impactos e a influência do aspecto humano, na melhoria de processos de software [Montoni e Rocha 2011], esse trabalho investigou o conceito de Endomarketing e a sua contribuição em iniciativas nessa área. 
Para tanto, esse estudo teve por objetivo identificar e analisar os principais instrumentos de Endomarketing presentes e importantes na melhoria de processos de software, a partir da percepção de profissionais envolvidos nessa área, o qual pode ser representado pela seguinte questão de pesquisa: Quais os instrumentos de Endomarketing presentes e importantes na melhoria de processos de software, segundo a percepção de profissionais dessa área?

Nesse estudo foram identificados instrumentos de Endomarketing, a partir de uma revisão da literatura, que permitiram a construção de um instrumento de pesquisa, validado por procedimentos de pré-teste. Posteriormente, esse instrumento foi aplicado no cenário nacional, a fim de levantar a percepção de profissionais envolvidos na melhoria de processos sobre os principais instrumentos de Endomarketing presentes e importantes, em suas experiências.

Essa pesquisa se justifica e seus resultados podem contribuir para uma melhor compreensão e um possível tratamento da influência do fator humano, na melhoria de processos de software, pois a identificação desses instrumentos de Endomarketing pode facilitar o comprometimento e minimizar a resistência por parte de colaboradores da organização.

Além desse primeiro capítulo de introdução, o restante do artigo está organizado em mais quatro capítulos e as referências. O capítulo 2 apresenta os conceitos básicos e discute trabalhos relacionados. O capítulo 3 detalha aspectos metodológicos dessa pesquisa. O capítulo 4 trata da análise e apresenta os resultados obtidos, enquanto, o capítulo 5 apresenta considerações finais sobre esse trabalho.

\section{Fundamentação Teórica}

\subsection{Fatores Críticos de Sucesso na Melhoria de Processos de Software}

Apesar do aumento no número de organizações, que adotam modelos de referência para melhorar seus processos de desenvolvimento de software, o percentual de empresas que usam esses modelos em relação ao total de organizações desenvolvedoras de software existentes ainda é pequeno. Segundo estudos anteriores, entre os principais fatores influentes na melhoria de processos encontram-se fatores humanos, tais como a falta de motivação e de comprometimento e a resistência a mudanças por parte de membros da organização [Montoni e Rocha 2011], [Nasir, Ahmad e Hassan 2008], [Brietzke et al. 2007], [Niazi et al. 2006], [Brietzke e Rabelo 2006], [Baddoo 2001].

Percebe-se que os aspectos que impactam na melhoria de processos vão além de questões tecnológicas, englobando fatores humanos, que podem tanto contribuir com o sucesso de um programa de melhoria, quanto prejudicá-lo. Porém, os estudos quanto a essas questões não tecnológicas são pouco explorados pelos pesquisadores da área [Bertelsen 1997], [Seaman 1999], [Coleman e O'Connor 2007], [Adolph et al. 2008].

Robbins (2005) também apresenta alguns aspectos humanos relevantes no comportamento organizacional, tais como motivação, satisfação com o trabalho e estresse profissional. Todos esses aspectos podem ser denominados fatores críticos de sucesso e influenciam claramente na satisfação e no comprometimento dos colaboradores, numa organização. 


\subsection{Endomarketing}

O termo "Endomarketing" (endo, do grego éndon, que significa "para dentro") ou marketing interno foi utilizado pela primeira vez na década de 70, quando Saul Faingaus Bekin, então, gerente de produtos de uma grande organização viu a necessidade de resolver problemas, como comunicação e integração entre departamentos, não entendimento por parte dos colaboradores de suas próprias funções e desconhecimento dos objetivos da empresa como um todo.

$\mathrm{Na}$ ocasião, Bekin chegou à conclusão de que para solucionar tais problemas, ele deveria aplicar o marketing dentro da empresa. Após obter sucesso em sua experiência, Bekin patenteou o termo "Endomarketing", utilizando o mesmo processo de formação de palavras como "endodontia" e "endoscopia" [Bekin 2004].

Entende-se por Endomarketing como "ações gerenciadas de marketing eticamente dirigidas ao público interno das organizações" [Bekin 2004, p. 47]. Esse conceito pode ser considerado uma projeção das ações de marketing utilizadas pela organização, com os clientes externos, aplicadas aos seus colaboradores. No Quadro 1 sintetiza-se as diferentes definições encontradas para o conceito Endomarketing.

Quadro 1. Definições de Endomarketing.

\begin{tabular}{|l|l|}
\hline Autor & Definição \\
\hline Bekin (2004) & Ações gerenciadas de marketing dirigidas ao público interno. \\
\hline Brum (1998) & Conjunto de ações utilizadas para vender sua própria imagem a funcionários. \\
\hline $\begin{array}{l}\text { Cerqueira } \\
(1994)\end{array}$ & $\begin{array}{l}\text { São projetos e ações utilizadas por uma empresa para consolidar a base cultural do } \\
\text { comprometimento dos seus funcionários com o desenvolvimento adequado de suas } \\
\text { diversas tecnologias. }\end{array}$ \\
\hline Gronroos (2003) & Conjunto de ações e instrumentos que vendem uma ideia ao público interno. \\
\hline
\end{tabular}

Atualmente, existem constantes mudanças nas maneiras de trabalho das organizações, como mudanças de tecnologia e de padrões, principalmente, no universo da Tecnologia da Informação [SOFTEX 2012]. Nesse contexto, se os colaboradores não estiverem cientes dos objetivos da organização com tais modificações, todas essas mudanças podem gerar um impacto na rotina de trabalho dos mesmos, sendo que a primeira reação esboçada é a resistência [Bekin 2004].

O Endomarketing colabora com a divulgação da empresa para o público interno, uma vez que, dessa forma, os mesmos entenderão os objetivos da organização e qual sua importância para que esses objetivos sejam alcançados [Brum 2005]. Com a adoção de ações de Endomarketing, a organização mantém uma relação transparente com os seus colaboradores, deixando claros os objetivos a serem alcançados pela empresa como um todo, dando a oportunidade destes harmonizarem os seus próprios objetivos, com os objetivos da empresa [Bekin 2004].

Essa relação transparente e a possibilidade de alinhamento dos objetivos da empresa com o dos colaboradores permite maior comprometimento por parte destes com a organização [Cerqueira 1994]. Esse comprometimento que o Endomarketing busca também é ressaltado por Bekin (2004), quando cita um artigo escrito por Theodore Levitt, na década de 70 e afirma que se a empresa espera atingir seus objetivos com o cliente externo, precisa garantir que internamente todos os envolvidos estejam comprometidos com esses mesmos objetivos. Sendo assim, o Endomarketing deve 
preceder o marketing externo, pois o comprometimento dos colaboradores é essencial para garantir o sucesso com os clientes finais.

Gronroos (2003) complementa ao argumentar que o Endomarketing proporciona aos colaboradores satisfação com seu ambiente de trabalho, com os relacionamentos com seus colegas de trabalho e com seu empregador. Ocorre que o Endomarketing utiliza instrumentos, que auxiliam na obtenção e na manutenção do comprometimento dos colaboradores. Essas ferramentas e ações podem abordar programas de incentivo à educação, ao esporte e à atuação comunitária, programas de treinamento, de desenvolvimento, de motivação, de entretenimento, de planos de carreira, de reconhecimento, valorização do funcionário e de crescimento [Bekin 2004].

Percebe-se que existem alguns programas e desafios, como os existentes na área de qualidade e de melhoria de processos de software, que necessitam de um esforço maior do público interno, para que seja alcançado o sucesso [Brum 1998]. Por vezes, esse esforço é exigido, principalmente, de pessoas com cargo mais baixo na organização e que, muitas vezes, a alta direção não dedica a devida atenção, em termos de valorização, informação e estímulo.

Normalmente, as organizações em busca de melhoria de processos simplesmente traçam uma missão para seus colaboradores, como a obtenção de uma certificação ou avaliação até uma determinada data. Nesse cenário, provavelmente, os colaboradores até se dispõem a participar, porém, às vezes, esse "sim", não tem um significado verdadeiro, pois falta o comprometimento para dar energia e a motivação necessária, para se chegar aos resultados esperados [Brum 1998].

No contexto da área de Tecnologia da Informação existem estudos anteriores relacionados ao emprego de Endomarketing, como os de Ellwanger e Nunes (2009) e Brietzke et al. (2007). Nesses estudos, ações de Endomarketing foram usadas, na efetividade de políticas de segurança da informação e num projeto de melhoria de processos de software, respectivamente.

Diante disso, se verifica que o Endomarketing tem papel importante na melhoria de processos de software, pois além de apoiar na preparação de todos para a conquista desejada, leva em consideração a importância das pessoas envolvidas nesse processo estarem motivadas e comprometidas, sabendo dos benefícios que os resultados trarão para a organização e para si e que o sucesso depende de cada um que participa dessa iniciativa [Brum 1998].

\subsection{Endomarketing na Melhoria de Processos de Software}

Um programa de Endomarketing exige que instrumentos sejam utilizados pela organização para que o sucesso de qualquer desafio ou processo de mudança seja alcançado. Esses instrumentos têm por objetivo oferecer um grau satisfatório de informação para os colaboradores, educar as pessoas sobre a empresa e suas metas, evitando espaço para resistência e boatos [Brum 1998].

Ressalta-se também que os instrumentos de Endomarketing adotados na organização devem ter relação com seus objetivos e sua cultura. Esses instrumentos devem ser selecionados, de acordo com a realidade da organização e com a eficácia dos mesmos, em seu contexto [Brum 1998], [Bekin 2004]. 
Quadro 2. Instrumentos de Endomarketing na melhoria de processos de software

\begin{tabular}{|c|c|}
\hline Instrumento & Descrição \\
\hline Ambientação & $\begin{array}{l}\text { Preparação e decoração do ambiente interno em momentos e acontecimentos } \\
\text { importantes do projeto de melhoria de processos de software, bem como em } \\
\text { situações diárias dos colaboradores do projeto. }\end{array}$ \\
\hline Blogs Internos & $\begin{array}{l}\text { Blogs internos informativos, participativos e colaborativos, que promovam a } \\
\text { discussão e comunicação interna de assuntos relacionados ao projeto de } \\
\text { melhoria de processos de software. }\end{array}$ \\
\hline $\begin{array}{l}\text { Canais diretos entre } \\
\text { a direção e } \\
\text { funcionários }\end{array}$ & $\begin{array}{l}\text { Canais diretos de comunicação entre colaboradores, a alta direção e o } \\
\text { patrocinador do projeto de melhoria de processos de software, proporcionando } \\
\text { credibilidade, confiança, respeito e transparência. }\end{array}$ \\
\hline $\begin{array}{l}\text { Datas e atividades } \\
\text { festivas }\end{array}$ & $\begin{array}{l}\text { Comemoração e lembrança em datas importantes e festivas, como em atividades } \\
\text { e conquistas do projeto de melhoria de processos de software, com cartazes, } \\
\text { cartões assinados pela alta direção, confraternizações, jantares e mensagens. }\end{array}$ \\
\hline Grife interna & $\begin{array}{l}\text { Camisetas, moletons, bonés, botons, sacolas, adesivos, bolsas, chaveiros, } \\
\text { mochilas, entre outros artigos, que divulguem a marca e o slogan do projeto de } \\
\text { melhoria de processos de software. }\end{array}$ \\
\hline Intranet & $\begin{array}{l}\text { Disponibilização de informações importantes da organização e do projeto de } \\
\text { melhoria de processos de software na Intranet. }\end{array}$ \\
\hline Jornal Interno & $\begin{array}{l}\text { Jornal interno periódico que trabalha campanhas internas direcionadas aos } \\
\text { colaboradores sobre o projeto de melhoria de processos de software. Também } \\
\text { pode tratar sobre colaboradores, informações de equipes, datas festivas, } \\
\text { principais conquistas, charges divertidas sobre situações do cotidiano e do } \\
\text { projeto de melhoria de processos de software. }\end{array}$ \\
\hline $\begin{array}{l}\text { Manuais técnicos e } \\
\text { educativos }\end{array}$ & $\begin{array}{l}\text { Material de auxílio aos colaboradores, com questões técnicas do projeto de } \\
\text { melhoria de processos de software, numa linguagem clara e informações } \\
\text { representadas por figuras, tais como história em quadrinhos ou "documento } \\
\text { passaporte", para carregar no bolso. }\end{array}$ \\
\hline $\begin{array}{l}\text { Manual e/ou vídeo } \\
\text { de integração à } \\
\text { empresa }\end{array}$ & $\begin{array}{l}\text { Manuais ou vídeos com um pouco da história da empresa, missão, visão, } \\
\text { princípios, objetivos, estado atual, negócio, produtos e perfil dos funcionários, } \\
\text { além de informações a respeito do projeto de melhoria de processo de software. }\end{array}$ \\
\hline $\begin{array}{l}\text { Material de } \\
\text { acompanhamento do } \\
\text { projeto ou iniciativa }\end{array}$ & $\begin{array}{l}\text { Material de acompanhamento periódico da situação do projeto de melhoria de } \\
\text { processos de software, com informações adicionais, metas e objetivos atingidos } \\
\text { e a serem alcançados, entre outras informações. }\end{array}$ \\
\hline Memória & $\begin{array}{l}\text { Exposição em livros, quadros, fotografias, entre outros meios de acontecimentos } \\
\text { importantes na história da organização e relacionadas à iniciativas anteriores na } \\
\text { área de qualidade, motivando os colaboradores para os desafios do projeto de } \\
\text { melhoria de processos de software. }\end{array}$ \\
\hline $\begin{array}{l}\text { Painéis e cartazes } \\
\text { motivacionais }\end{array}$ & $\begin{array}{l}\text { Painéis e cartazes motivacionais com figuras, esquemas e cores para abordar de } \\
\text { forma leve e descontraída assuntos sérios, tais como mudanças provocadas pelo } \\
\text { projeto de melhoria, além de comemorações relacionadas ao projeto. }\end{array}$ \\
\hline Palestras internas & $\begin{array}{l}\text { Palestras de repasse para os demais colaboradores sobre conhecimento adquirido } \\
\text { em treinamentos externos, sobre assuntos técnicos e de progresso relacionados } \\
\text { ao projeto de melhoria de processos de software, como divulgação de resultados, } \\
\text { estado atual e próximas metas. }\end{array}$ \\
\hline $\begin{array}{c}\text { Reuniões com } \\
\text { videoconferências } \\
\text { ou webconferências }\end{array}$ & $\begin{array}{l}\text { Reuniões ou encontros virtuais referentes ao projeto de melhoria de processos de } \\
\text { software, permitindo uma aproximação e facilitação na comunicação entre os } \\
\text { envolvidos e/ou convidados localizados em diferentes locais. }\end{array}$ \\
\hline $\begin{array}{l}\text { Valorização de } \\
\text { funcionários }\end{array}$ & $\begin{array}{l}\text { Valorização de colaboradores envolvidos e em destaque no projeto de melhoria } \\
\text { de processos de software por meio de incentivos financeiros, promoções, troféus, } \\
\text { medalhas, homenagens, utilidades domésticas, eletrônicos, entre outros. }\end{array}$ \\
\hline $\begin{array}{l}\text { Vídeos informativos } \\
\text { e motivacionais }\end{array}$ & $\begin{array}{l}\text { Vídeos informativos e motivacionais utilizados no lançamento do projeto de } \\
\text { melhoria de processos de software, de novos desafios, conquistas ou no } \\
\text { encerramento do projeto. }\end{array}$ \\
\hline
\end{tabular}


Embora, não se tenha identificado um estudo quantitativo anterior relacionado ao tema dessa pesquisa, observa-se que parte dos instrumentos comumente utilizados em programas de Endomarketing podem ser aplicados para fins de motivação e comprometimento de colaboradores, na melhoria de processos de software.

Nessa pesquisa foram selecionadas de forma qualitativa instrumentos de Endomarketing, baseados em Brum (1998), Bekin (2004), Brietzke et al. (2007) e Ellwanger (2009), conforme Quadro 2. Para essa seleção foi conduzida uma avaliação individual dos instrumentos encontrados na revisão teórica, que apresentavam potencial de uso e de adaptação à realidade da indústria de software e de iniciativas em melhoria de processos de software, tendo em vista que nem todos os instrumentos identificados nessa revisão são aplicáveis, em quaisquer contextos.

A descrição dos instrumentos selecionados e apresentada na segunda coluna do Quadro 2, também foi adaptada pelos autores ao contexto da melhoria de processos de software. Depois, esses instrumentos foram usados como base na elaboração e na validação do instrumento de pesquisa adotado, numa pesquisa survey, conforme se detalha no próximo capítulo desse artigo.

\section{Metodologia de Pesquisa}

Essa pesquisa divide-se em duas partes, com características distintas. A primeira se caracteriza como de natureza exploratória e de caráter qualitativo. Gil (2010) define que pesquisas exploratórias têm por objetivo proporcionar maior familiaridade com o problema, com vistas a torná-lo mais explícito ou a construir hipóteses. Essa primeira etapa teve por finalidade identificar instrumentos de Endomarketing, que poderiam contribuir na melhoria de processos de software, assegurando comprometimento por parte dos colaboradores. Para tanto foi realizada uma revisão da literatura, abrangendo livros e artigos de periódicos e de eventos nacionais e internacionais sobre melhoria de processos de software e Endomarketing.

Já a segunda parte da pesquisa possui natureza descritiva e caráter quantitativo. Segundo Gil (2010), uma pesquisa descritiva tem por objetivo descrever características de determinada população ou o estabelecimento de relações entre variáveis. Nessa etapa foi gerada uma versão preliminar do instrumento de pesquisa, a partir do conjunto inicial de instrumentos de Endomarketing levantados na primeira parte dessa pesquisa.

Destaca-se que as variáveis referentes à avaliação de presença e de importância dos instrumentos de Endomarketing na melhoria de processos foram mensuradas, por meio de uma escala Likert de cinco pontos [Hair et al. 2005], com opções de 1 ("Presença fraca ou nenhuma") a 5 ("Muito presente"), para a avaliação de presença e, de 1 ("Importância fraca ou nenhuma") a 5 ("Muito Importante"), para a avaliação de importância. Sendo que, em ambas as avaliações existia a opção na escala de "SCO" (Sem Condições de Opinar). Já na parte do instrumento referente à caracterização do perfil dos respondentes, as questões basearam-se em Brietzke e Rabelo (2006) e Nasir, Ahmad e Hassan (2008).

Antes da coleta de dados, o instrumento de pesquisa foi submetido a procedimentos de pré-teste de especialistas e de respondentes, para fins de validação das variáveis a serem analisadas, assim como a aplicabilidade, clareza, legibilidade, 
layout adequado e solidez teórica das questões [Cooper e Schindler 2003]. No pré-teste de especialistas, quatro especialistas participaram de forma presencial e remota (e-mail e videoconferência), sendo dois deles, com vasta experiência em melhoria de processos de software e os outros dois, com grande vivência em Endomarketing. Destaca-se que nesse pré-teste, um dos especialistas envolvidos foi o autor do termo e da principal referência adotada nesse trabalho, em Endomarketing.

Após a conclusão do pré-teste de especialistas, uma nova versão do instrumento foi gerada, contemplando as melhorias julgadas pertinentes e relevantes pelos pesquisadores: melhoria na contextualização do respondente, na página inicial do questionário; inclusão de variáveis sócio-demográficas referentes ao porte e natureza da organização do respondente; inclusão do instrumento de Endomarketing "Blogs Internos". Essa nova versão foi então submetida ao segundo pré-teste, com doze respondentes, que também apontaram melhorias, contempladas na versão final do instrumento, utilizada na etapa de coleta da survey: apresentação dos instrumentos de Endomarketing, por ordem alfabética; melhoria na descrição e explicação de alguns dos instrumentos de Endomarketing.

A coleta de dados foi realizada no período de 27 de agosto a 03 de novembro de 2013, por meio de questionário eletrônico disponibilizado no software Qualtrics: https://qtrial.qualtrics.com/SE/?SID $=S V$ _tSuq9iQwvicgNn. Como a pesquisa teve por objetivo levantar a presença e a importância dos instrumentos de Endomarketing, segundo a percepção de profissionais, que têm ou tiveram experiência na melhoria de processos de software, em nível nacional, a divulgação da pesquisa ocorreu por meio de redes sociais, rede de contatos dos pesquisadores, publicação de mensagens em comunidades e páginas de grupos de especialistas no Facebook, Twitter e LinkedIn, além de contatos diretos por e-mail.

Em torno de 300 profissionais foram contatados e muitos desses foram encontrados no site do programa MPS.BR [SOFTEX 2014]. Ainda, conforme a estratégia de divulgação adotada, após um período de três semanas, como muitos dos profissionais convidados não retornaram ao primeiro contato, o pedido de participação na pesquisa foi reforçado, através dos canais supracitados. Além disso, outro contato foi realizado sete dias antes do término do período de coleta de dados da pesquisa.

Obteve-se um total de 88 questionários iniciados, sendo que 48 foram concluídos e considerados válidos para fins de análise, conforme critérios de descarte adotados: questionários incompletos ou que usaram apenas um ponto da escala para todos os instrumentos de Endomarketing. Em relação ao motivo do alto índice de abandono pode ter se dado pela falta de disponibilidade dos respondentes ou, como muitos reportaram pela insegurança, por não conhecerem em profundidade a área de Endomarketing.

$\mathrm{Na}$ análise, com o objetivo de organizar e sintetizar os dados, possibilitando estabelecer respostas e conclusões ao problema e objetivos dessa pesquisa, a partir dos dados obtidos [Hoppen, Lapointe, Moreau 1996] [Gil 2009] foram utilizadas técnicas e procedimentos de estatística [Hair et al. 2005], com o auxílio de planilha eletrônica, Microsoft Excel 2007, conforme se apresenta no próximo capítulo. 


\section{Análise dos resultados}

Inicialmente, esse capiítulo apresenta o perfil dos respondentes, por meio de análise descritiva e após, as análises referentes à presença e importância dos instrumentos de Endomarketing, no contexto da melhoria de processos de software.

\subsection{Perfil dos respondentes}

Os respondentes foram caracterizados pelos critérios de faixa etária, estado de residência, nível de formação acadêmica, atuação na universidade, indústria de software e em projetos de melhoria de processos de software, quantidade de projetos e tempo de atuação nessa área e em desenvolvimento de software, além de porte e natureza das organizalções, onde tiveram experiências na melhoria de processos de software.

De modo geral, a análise descritiva mostra um perfil dos respondentes, com as seguintes características:

- média de idade dos respondentes de 35 anos, sendo que 42 dos 48 profissionais possuem mais que 31 anos de idade e nenhum é mais jovem, que 25 anos;

- residentes em sua maioria nas regiões Sudeste (22) e Sul (16), tendo maior frequência nos estados do Rio de Janeiro, Rio Grande do Sul e São Paulo;

- alto nível de formação acadêmica, pois 44 dos 48 respondentes possuem doutorado (8), mestrado (25) e especialização (11);

- participação ativa na universidade, seja como professor (28), pesquisador (10), aluno (16) ou consultor (15), o que representa um alto nível de conhecimento dos mesmos;

- alto nível de maturidade em desenvolvimento de software (42 dos 48 respondentes possuem mais de 8 anos de experiência), sendo a maioria como gerente de projetos, analista de qualidade, desenvolvedor de software, analista de sistemas e ainda, 29 dos respondentes citaram outras atuações, tais como diretor, consultor e gerente de configuração;

- metade dos respondentes possuem mais de 8 anos de experiência em melhoria de processos de software, atuando como implementador, consultor, membro de equipe de melhoria de processos de software e de qualidade;

- alta experiência com projetos na área de melhoria de processos, pois metade dos respondentes participaram de mais de 10 projetos, em média em 7,8 projetos;

- maior parte dos respondentes com experiências em micro (19) e pequenas (38) empresas, mas também com experiência em média (28) e grande (20) empresas;

- predominância de respondentes com experiências em empresas privadas (47), visto que todos, com exceção de um, assinalaram essa opção e apenas 17 , com experiência em empresas públicas.

Percebe-se pela caracterização do perfil dos respondentes, que os profissionais da amostra são de fato especialistas na área, pois possuem alto nível de conhecimento, formação e de vivência prática na melhoria de processos de software e desenvolvimento de software, com participação efetiva na academia e na indústria. 


\subsection{Análise da presença dos instrumentos de Endomarketing}

No Quadro 3 são apresentados os resultados obtidos em relação à presença do Endomarketing na melhoria de processos de software, com a média e o desvio padrão para cada um dos seus instrumentos.

\section{Quadro 3. Presença dos instrumentos de Endomarketing}

\begin{tabular}{|l|c|c|c|c|c|c|c|}
\hline \multirow{2}{*}{ Instrumentos de Endomarketing } & \multicolumn{5}{|c|}{ Avaliação da Presença } \\
\cline { 2 - 8 } & $\mathbf{1}$ & $\mathbf{2}$ & $\mathbf{3}$ & $\mathbf{4}$ & $\mathbf{5}$ & Média & D. Padrão \\
\hline Intranet & 2 & 4 & 9 & 13 & 19 & $\mathbf{3 , 9 1}$ & 1,16 \\
\hline Palestras internas & 3 & 2 & 14 & 12 & 17 & $\mathbf{3 , 7 9}$ & 1,17 \\
\hline Canais diretos entre a direção e funcionários & 4 & 10 & 6 & 10 & 17 & $\mathbf{3 , 5 5}$ & 1,40 \\
\hline Manuais técnicos e educativos & 9 & 9 & 9 & 10 & 11 & $\mathbf{3 , 1 0}$ & 1,45 \\
\hline Material de acompanhamento do projeto ou iniciativa & 5 & 12 & 12 & 11 & 8 & $\mathbf{3 , 1 0}$ & 1,26 \\
\hline Valorização de funcionários & 9 & 14 & 12 & 4 & 9 & 2,79 & 1,37 \\
\hline Blogs Internos & 9 & 14 & 10 & 4 & 9 & 2,78 & 1,40 \\
\hline Ambientação & 10 & 13 & 8 & 7 & 7 & 2,73 & 1,39 \\
\hline Datas e atividades festivas & 11 & 12 & 8 & 11 & 5 & 2,72 & 1,35 \\
\hline Painéis e cartazes motivacionais & 9 & 16 & 10 & 6 & 7 & 2,71 & 1,32 \\
\hline Jornal Interno & 11 & 17 & 4 & 10 & 6 & 2,65 & 1,38 \\
\hline Manual e/ou vídeo de integração à empresa & 10 & 18 & 6 & 5 & 8 & 2,64 & 1,39 \\
\hline Reuniões com videoconferências ou webconferências & 18 & 8 & 8 & 6 & 7 & 2,49 & 1,49 \\
\hline Grife interna & 17 & 12 & 5 & 8 & 5 & 2,40 & 1,41 \\
\hline Memória & 18 & 14 & 3 & 5 & 5 & 2,22 & 1,38 \\
\hline Vídeos informativos e motivacionais & 20 & 13 & 3 & 2 & 7 & 2,18 & 1,45 \\
\hline
\end{tabular}

Apesar de alguns instrumentos se destacarem, com maiores médias de presença, nenhum deles ultrapassou 4 pontos de média, mostrando que existe heterogeneidade na perceção de presença dos instrumentos de Endomarketing, na melhoria de processos de software. A partir dessa diversidade da amostra observa-se a ausência de um padrão de instrumentos adotados, para comprometer os colaboradores, nas iniciativas nessa área.

Entre os instrumentos de maior média de presença na melhoria de processos destacam-se: "Intranet" $(3,91)$, "Palestras internas" $(3,79)$ e "Canais diretos entre a direção e funcionários" $(3,55)$. Esses instrumentos mostram-se com maior popularidade, para conquista de comprometimento de colaboradores, na experiência dos respondentes.

Os instrumentos "Material de acompanhamento do programa" $(3,10)$ e "Manuais técnicos e educativos" $(3,10)$ também obtiveram média superior a 3 pontos, mostrando uma boa presença na experiências de melhoria de processos de software. Já, quando analisa-se os instrumentos, com menor presença destacam-se: "Vídeos informativos e motivacionais" $(2,18)$, "Memória" $(2,22)$, "Grife interna" $(2,40)$ e "Reuniões com vídeo conferência ou webconferências" $(2,49)$.

\subsection{Análise da importância dos instrumentos de Endomarketing}

Conforme Quadro 4, em relação à importância dos instrumentos de Endomarketing na melhoria de processos de software destaca-se maior homogeneidade nas respostas dos respondentes, do que na avaliação da presença (Quadro 3).

No Quadro 4, dois instrumentos de Endomarketing foram considerados essenciais na melhoria de processos de software, devido à unanimidade das respostas dessa amostra: "Canais diretos entre a direção e funcionários" e "Intranet". Isso se deve 
pela alta média alcançada $(4,83$ e 4,81 , respectivamente) e baixo desvio padrão $(0,43$ e $0,45)$, mostrando que a dispersão de opiniões foi extremamente pequena, nesses dois casos. Seguindo esse raciocínio, os instrumentos "Palestras Internas" e "Valorização de funcionários" aparecem na sequência, com uma dispersão um pouco maior $(0,71$ e 1,05, respectivamente), mas também com médias elevadas (4,60 e 4,27, respectivamente).

Quadro 4. Importância dos instrumentos de Endomarketing

\begin{tabular}{|l|c|c|c|c|c|c|c|c|}
\hline \multirow{2}{*}{ Instrumentos de Endomarketing } & \multicolumn{5}{c|}{ Avaliaço da Importância } \\
\cline { 2 - 8 } & $\mathbf{1}$ & $\mathbf{2}$ & $\mathbf{3}$ & $\mathbf{4}$ & $\mathbf{5}$ & Média & D. Padrão \\
\hline Canais diretos entre a direção e funcionários & 0 & 0 & 1 & 6 & 40 & $\mathbf{4 , 8 3}$ & $\mathbf{0 , 4 3}$ \\
\hline Intranet & 0 & 0 & 1 & 7 & 40 & $\mathbf{4 , 8 1}$ & $\mathbf{0 , 4 5}$ \\
\hline Palestras internas & 0 & 1 & 3 & 10 & 34 & $\mathbf{4 , 6 0}$ & 0,71 \\
\hline Valorização de funcionários & 1 & 4 & 3 & 13 & 27 & $\mathbf{4 , 2 7}$ & 1,05 \\
\hline Material de acompanhamento do projeto ou iniciativa & 1 & 1 & 11 & 15 & 20 & $\mathbf{4 , 0 8}$ & 0,96 \\
\hline Manuais técnicos e educativos & 1 & 3 & 9 & 14 & 21 & $\mathbf{4 , 0 6}$ & 1,04 \\
\hline Blogs Internos & 1 & 3 & 7 & 20 & 16 & $\mathbf{4 , 0 0}$ & 0,98 \\
\hline Painéis e cartazes motivacionais & 2 & 4 & 10 & 22 & 10 & 3,71 & 1,03 \\
\hline Reuniões com videoconferências ou webconferências & 3 & 3 & 13 & 13 & 14 & 3,70 & 1,17 \\
\hline Datas e atividades festivas & 4 & 3 & 10 & 16 & 13 & 3,67 & 1,21 \\
\hline Manual e/ou vídeo de integração à empresa & 1 & 7 & 13 & 15 & 12 & 3,63 & 1,08 \\
\hline Vídeos informativos e motivacionais & 2 & 8 & 13 & 11 & 12 & 3,50 & 1,19 \\
\hline Ambientação & 4 & 5 & 10 & 17 & 9 & 3,49 & 1,20 \\
\hline Jornal Interno & 4 & 8 & 8 & 17 & 10 & 3,45 & 1,25 \\
\hline Memória & 7 & 9 & 11 & 8 & 10 & 3,11 & 1,39 \\
\hline Grife interna & 9 & 7 & 19 & 6 & 6 & 2,85 & 1,25 \\
\hline
\end{tabular}

Na avaliação de importância, as respostas tiveram predominância entre as opções 4 e 5, na escala adotada, mostrando forte concordância na percepção dos respondentes. Nesse sentido, outros instrumentos também tiveram boas médias: "Material de acompanhamento do projeto ou iniciativa" $(4,08)$, "Manuais técnicos e educativos" $(4,06)$ e "Blogs internos" $(4,00)$.

Entre os instrumentos avaliados, com menor importância encontram-se: "Grife Interna" (2,85), "Memória" (3,11), "Jornal Interno" (3,45) e "Ambientação" $(3,49)$. Porém, apenas em função dessas médias, não se pode afirmar que tais instrumentos não são importantes, visto que apenas um entre todos obteve média abaixo de 3 .

No que tange à avaliação da importância observa-se que os instrumentos de Endomarketing identificados e selecionados na revisão teórica e, posteriormente, validados no instrumento de pesquisa pelos procedimentos de pré-teste podem ser considerados como eficazes, em relação às variáveis a serem mensuradas e analisadas, no contexto da melhoria de processos de software.

\section{Análise comparativa entre presença e importância}

As seções anteriores fizeram uma análise da presença e da importância dos instrumentos de Endomarketing de forma isolada. A análise dessa seção mostra um diagnóstico da situação atual de presença dos instrumentos validados como importantes nesse trabalho, no cenário nacional.

No Quadro 5 são comparadas as médias e os desvios padrões obtidos para as variáveis validadas como importantes, com as médias e os desvios padrões da presença dessas variáveis. A coluna "Gap", basicamente, mostra a lacuna entre o que é 
considerado importante pelos respondentes, mas não está presente, ou seja, o que está faltando ser adotado nos projetos de melhoria de processos de software. Portanto, essas lacunas podem ser tratadas como oportunidades de melhoria e desafios de gestão da qualidade e de uso do Endomarketing, nesse contexto.

Quadro 5. Presença dos instrumentos de Endomarketing em relação à importância

\begin{tabular}{|l|c|c|c|c|c|}
\hline \multirow{2}{*}{ Instrumentos de Endomarketing } & \multicolumn{2}{c|}{ Importância } & \multicolumn{2}{c|}{ Presença } & \multirow{2}{*}{ Gap } \\
\cline { 2 - 5 } & Média & D. Padrão & Média & D. Padrão & \\
\hline Ambientação & 3,49 & 1,20 & 2,73 & 1,39 & 0,76 \\
\hline Blogs Internos & $\mathbf{4 , 0 0}$ & 0,98 & $\mathbf{2 , 7 8}$ & 1,40 & $\mathbf{1 , 2 2}$ \\
\hline Canais diretos entre a direção e funcionários & $\mathbf{4 , 8 3}$ & 0,43 & $\mathbf{3 , 5 5}$ & 1,40 & $\mathbf{1 , 2 8}$ \\
\hline Datas e atividades festivas & 3,67 & 1,21 & 2,72 & 1,35 & 0,95 \\
\hline Grife interna & 2,85 & 1,25 & 2,40 & 1,41 & 0,45 \\
\hline Intranet & $\mathbf{4 , 8 1}$ & 0,45 & $\mathbf{3 , 9 1}$ & 1,16 & 0,90 \\
\hline Jornal Interno & 3,45 & 1,25 & 2,65 & 1,38 & 0,80 \\
\hline Manuais técnicos e educativos & $\mathbf{4 , 0 6}$ & 1,04 & $\mathbf{3 , 1 0}$ & 1,45 & 0,96 \\
\hline Manual e/ou vídeo de integração à empresa & 3,63 & 1,08 & 2,64 & 1,39 & 0,99 \\
\hline Material de acompanhamento do projeto ou iniciativa & $\mathbf{4 , 0 8}$ & 0,96 & $\mathbf{3 , 1 0}$ & 1,26 & $\mathbf{0 , 9 8}$ \\
\hline Memória & 3,11 & 1,39 & 2,22 & 1,38 & 0,89 \\
\hline Painéis e cartazes motivacionais & 3,71 & 1,03 & 2,71 & 1,32 & 1,00 \\
\hline Palestras internas & $\mathbf{4 , 6 0}$ & 0,71 & $\mathbf{3 , 7 9}$ & 1,17 & 0,81 \\
\hline Reuniões com videoconferências ou webconferências & 3,70 & 1,17 & 2,49 & 1,49 & 1,21 \\
\hline Valorização de funcionários & $\mathbf{4 , 2 7}$ & 1,05 & $\mathbf{2 , 7 9}$ & 1,37 & $\mathbf{1 , 4 8}$ \\
\hline Vídeos informativos e motivacionais & 3,50 & 1,19 & 2,18 & 1,45 & 1,32 \\
\hline
\end{tabular}

No Quadro 5, destaca-se que os instrumentos de Endomarketing considerados importantes pelos respondentes "Valorização de funcionários" e "Canais diretos entre a direção e funcionários" possuem os maiores valores de Gap, quando se analisa a presença desses instrumentos, na melhoria de processos de software $(1,48$ e 1,28, respectivamente). Isso demonstra que os benefícios e os efeitos referentes ao comprometimento, que podem ser obtidos por esses instrumentos no tratamento do fator humano poderiam ser melhor aproveitados, na gestão e na condução de iniciativas de melhoria de processos de software, no cenário brasileiro.

Esse mesmo raciocínio pode ser empregado para outros instrumentos de Endomarketing considerados importantes pelos respondentes, como "Blogs internos" e "Material de acompanhamento do projeto ou iniciativa", que também obtiveram altos índices de Gap (1,22 e 0,98, respectivamente). Portanto, a partir do conteúdo do Quadro 5 e de modo geral percebe-se que em todos os casos, os instrumentos possuem menor presença do que deveriam ter, segundo o grau de importância apontado pelos respondentes dessa amostra.

Por fim, reconhece-se que diversas outras análises podem ser feitas considerando o conteúdo do Quadro 5 (assim como, dos quadros 4 e 3 supracitados), sendo essa a maior contribuição desse estudo, na área de melhoria de processos de software.

\subsection{Análise comparativa entre a visão interna e externa}

Além das análises individuais e comparativas entre a presença e a importância dos instrumentos de Endomarketing, na melhoria de processos de software foi realizada uma análise comparativa entre a visão dos respondentes, que atuam em papéis dentro das 
organizações, denominada nesse estudo de "visão interna" e dos respondentes, que possuem atuação fora das organizações, denominada de "visão externa".

Essa análise justifica-se pela eliminação da possibilidade de algum viés, nos resultados obtidos nessa pesquisa. Para tanto, as atuações em projetos de melhoria de processos de software foram divididas em: i) visão interna: patrocinador, membro de equipe de qualidade, de melhoria de processos de software e de projeto de desenvolvimento de software; ii) visão externa: consultor e membro de equipe de implementação (implementador).

No Quadro 6 são apresentadas as médias da visão geral (MG, todos os respondentes), interna (MI) e externa (ME), além do desvio padrão de cada avaliação, possibilitando uma comparação entre os resultados dessas visões.

Quadro 6. Análise Comparativa entre as diferentes visões

\begin{tabular}{|l|c|c|c|c|c|c|c|c|}
\hline \multirow{2}{*}{\multicolumn{1}{|c|}{ Instrumentos de Endomarketing }} & Importância & \multicolumn{4}{c|}{ Presença } \\
\cline { 2 - 9 } & MG & MI & ME & $\begin{array}{c}\text { Desvio } \\
\text { Padrão } \\
\text { Geral }\end{array}$ & MG & MI & ME & $\begin{array}{c}\text { Desvio } \\
\text { Padrão } \\
\text { Geral }\end{array}$ \\
\hline Ambientação & 3,49 & 3,50 & 3,55 & 1,20 & 2,73 & 2,50 & 2,50 & 1,39 \\
\hline Blogs Internos & 4,00 & 3,62 & 4,30 & 0,98 & 2,78 & 1,75 & 3,50 & 1,40 \\
\hline $\begin{array}{l}\text { Canais diretos entre a direção e } \\
\text { funcionários }\end{array}$ & 4,83 & 5,00 & 4,56 & 0,43 & 3,55 & 4,37 & 2,89 & 1,40 \\
\hline Datas e atividades festivas & 3,67 & 3,62 & 4,00 & 1,21 & 2,72 & 2,75 & 2,78 & 1,35 \\
\hline Grife interna & 2,85 & 2,50 & 3,10 & 1,25 & 2,40 & 2,12 & 2,11 & 1,41 \\
\hline Intranet & 4,81 & 4,75 & 4,80 & 0,45 & 3,91 & 3,28 & 4,10 & 1,16 \\
\hline Jornal Interno & 3,45 & 2,50 & 3,50 & 1,25 & 2,65 & 2,50 & 3,00 & 1,38 \\
\hline Manuais técnicos e educativos & 4,06 & 3,75 & 4,00 & 1,04 & 3,10 & 2,87 & 3,20 & 1,45 \\
\hline Manual e/ou vídeo de integração à empresa & 3,63 & 3,50 & 3,30 & 1,08 & 2,64 & 2,75 & 2,33 & 1,39 \\
\hline $\begin{array}{l}\text { Material de acompanhamento do projeto } \\
\text { ou iniciativa }\end{array}$ & 4,08 & 4,12 & 4,20 & 0,96 & 3,10 & 3,25 & 3,20 & 1,26 \\
\hline Memória & 3,11 & 3,37 & 3,00 & 1,39 & 2,22 & 2,25 & 1,75 & 1,38 \\
\hline Painéis e cartazes motivacionais & 3,71 & 3,12 & 4,10 & 1,03 & 2,71 & 2,00 & 3,20 & 1,32 \\
\hline Palestras internas & 4,60 & 4,75 & 4,70 & 0,71 & 3,79 & 4,00 & 3,70 & 1,17 \\
\hline $\begin{array}{l}\text { Reuniões com videoconferências ou } \\
\text { webconferências }\end{array}$ & 3,70 & 3,57 & 3,88 & 1,17 & 2,49 & 1,87 & 2,67 & 1,49 \\
\hline Valorização de funcionários & 4,27 & 3,37 & 4,40 & 1,05 & 2,79 & 2,62 & 2,90 & 1,37 \\
\hline Vídeos informativos e motivacionais & 3,50 & 3,12 & 3,80 & 1,19 & 2,18 & 2,12 & 1,87 & 1,45 \\
\hline
\end{tabular}

Ao todo, 10 respondentes se caracterizaram na visão externa e 8 na visão interna. Observa-se, no Quadro 6, que de modo geral mantêm-se a mesma percepção sobre a presença e importância dos instrumentos de Endomarketing, na melhoria de processos de software. Evidencia-se que as visões interna e externa estão de acordo, com o desvio padrão geral de cada um dos instrumentos de Endomarketing. Essa análise torna-se importante, na medida em que clareia e evidencia a concordância entre as visões interna e externa, potencializando os resultados gerais apresentados nessa pesquisa.

\subsection{Percepção geral dos instrumentos de Endomarketing}

No questionário havia também uma questão aberta, para que os respondentes pudessem acrescentar outros instrumentos de Endomarketing observados em suas experiências na melhoria de processos de software. Essas respostas foram analisadas qualitativamente e no total, nove outros instrumentos foram levantados: dinâmicas em grupo, treinamentos, 
reuniões periódicas, jogos, perguntas mais frequentes, envio de e-mails sobre as melhorias realizadas nos processos, semana da qualidade e estímulo à participação e ao compartilhamento de decisões.

Esses nove instrumentos podem vir a ser incorporadas em futuras versões do instrumento de pesquisa, conforme nova revisão de literatura e validação com especialistas, que venham a evidenciar os mesmos como essenciais, na melhoria de processos de software. Posteriormente, podem ser validados em futura replicação dessa pesquisa ou em futuras pesquisas sobre o tema, assim como podem ser levados em consideração para fins de adoção e tratamento por gestores de iniciativas nessa área.

Esse capítulo apresentou a análise dos dados, com enfoque na etapa quantitativa da pesquisa, onde foi conduzida uma survey. O próximo capítulo concebe considerações finais, enfatizando contribuições e sugerindo trabalhos futuros relacionados ao tema.

\section{Conclusão}

Este estudo abordou dois temas distintos, o Endomarketing e a melhoria de processos de software, associando esses dois temas, tornando-o relevante e com contribuição científica importante, na área de melhoria de processos de software.

Destaca-se que dificilmente, uma organização adotará todos os instrumentos de Endomarketing apresentados nesse estudo, pois isso requer tempo, custos e adequação à realidade e às necessidades. Por outro lado, o estudo mostra que a seleção e a combinação de parte desses instrumentos, na melhoria de processos de software podem beneficiar e facilitar o alcance e o comprometimento por parte dos colaboradores, com os objetivos finais, nessa área.

Com a conclusão dessa pesquisa observa-se que seu objetivo principal foi alcançado, quando mapeia e analisa os principais instrumentos de Endomarketing presentes e importantes na melhoria de processos de software, a partir da percepção de profissionais dessa área. Portanto, apresenta uma possível resposta para o problema de pesquisa identificado e que norteou todo o desenvolvimento desse estudo.

No que tange às contribuições acadêmicas, esse estudo analisou uma lacuna de investigação referente à adoção do Endomarketing, na melhoria de processos de software. Também contribuiu com um instrumento pesquisa, capaz de mensurar instrumentos de Endomarkenting nessa área, podendo ser reutilizado em pesquisas futuras, bem como, replicado em outros contextos, como em outros países, por exemplo.

No que tange à contribuição gerencial em melhoria de processos de software, os resultados dessa pesquisa podem auxiliar profissionais de organizações e especialistas nessa área, no tratamento da influência do fator humano, utilizando como base o conjunto de instrumentos de Endomarketing identificados nesse estudo.

Percebe-se que a presença atual dos instrumentos de Endomarketing na melhoria de processos de software é baixa, se comparada com a sua importância, na opinião dos respondentes dessa amostra. Muitos instrumentos considerados importantes pelos especialistas tiveram média de presença abaixo da média de importância. Isso mostra que as organizações precisam rever suas estratégias, a gestão do fator humano e os métodos de implementação de iniciativas nessa área, uma vez que esses instrumentos 
podem facilitar o processo de comprometimento e motivação dos colaboradores envolvidos, bem como influenciar, no resultado final dessas iniciativas.

O estudo apresenta a atual presença em relação à importância de cada instrumento de Endomarketing, na percepção dos respondentes dessa amostra da pesquisa, que pela caracterização do perfil podem vir a ser considerados especialistas e com atuação efetiva na área de melhoria de processos de software.

Sugere-se como um possível trabalho futuro e continuidade dessa pesquisa, a aplicação dos instrumentos de Endomarketing considerados mais importantes nesse estudo, numa determinada organização, por meio de um estudo de caso, para aprofundar, validar e relatar os resultados obtidos com esses instrumentos de uma maneira prática.

Por fim, como esse estudo foi aplicado no contexto brasileiro, com divulgação e coleta abrangendo todas as regiões do país, se sugere como trabalho futuro, a replicação dessa pesquisa em outros contextos, como em outros países, para fins de comparação dos resultados, análise da influência da cultura, além de validação do instrumento de pesquisa construído nessa pesquisa.

\section{Referências}

Adolph, S., Hall, W. e Kruchten, P. (2008) "A methodological leg to stand on: Lessons learned using grounded theory to study software development", pp. IBM Toronto Software Lab.; IBM Centers for Advanced Studies, CAS, Richmond Hill, ON, Canada.

Baddoo, N. (2001) "Motivators and de-motivators in software process improvement: an empirical study", $\mathrm{PhD}$, University of Hertfordshire, UK.

Bertelsen, O.W. (1997) "Toward a unified field of SE research and practice”, IEEE Software, v. 14, n. 6, p. 87-88.

Bekin, S. F. (2004) "Endomarketing: como praticá-lo com sucesso". São Paulo: Prentice-Hall.

Brum, A. de M. (2005) "Face a Face com o Endomarketing. O Papel Estratégico das Lideranças no Processo das Informações”. Porto Alegre: L\&PM.

Brum, A. M. (1998) "Endomarketing como Estratégia de Gestão: Encante seu Cliente Interno". Porto Alegre: L\&PM.

Brietzke, J., López, P. A. do P., ALBERTUNI, I. e Richter, L. A.. (2007) “A Conquista do MPS.BR Nível F na Qualità Informática: Um Caso de Sucesso”, In: VI Simpósio Brasileiro de Qualidade de Software, Pernambuco, Brasil.

Brietzke, J.; Rabelo, A. (2006) "Resistance Factors in Software Process Improvement", CLEI Eletronic Journal, v. 9, n. 1. p. 4.

Cerqueira, W. (1994) "Endomarketing: educação e cultura para a qualidade". Rio de Janeiro: Qualitymark.

Coleman, G. e O'Connor, R. (2007) "Using grounded theory to understand software process improvement: a study of Irish software product companies, Information and Software Technology", v. 49. 
Cooper, D.; Schindler, P. (2003) "Método de pesquisa em Administração". 7ed. Porto Alegre: Bookman.

Dessler, G. (2002) "Human Resource Management. Upper Saddle River”, NJ: Prentice Hall, 653p.

Ellwanger, C. e Nunes, R. C. (2009) "Impacto da Utilização de Técnicas de Endomarketing na Efetividade de uma Política de Segurança da Informação".

Gil, A. C. (2010) “Como elaborar projetos de pesquisa”. 5 Edição. São Paulo: Atlas.

Gronroos, C. (2003) "Marketing: gerenciamento e serviços". 2. ed. Rio de Janeiro: Elsevier.

Hair, J. F. et al. (2009) “Análise multivariada de dados”. Porto Alegre: Bookman.

Hoppen, N.; Lapointe, L.; Moreau, E. (1996) "Um guia para avaliação de artigos de pesquisa em sistemas de informação". Revista Eletrônica de Administração, Porto Alegre, 3ed., v. 2, n. 2.

Montoni, M. A. e Rocha, A. R. C. (2011) "Uma Investigação sobre os Fatores Críticos de Sucesso em Iniciativas de Melhoria de Processos de Software”. In: X Simpósio Brasileiro de Qualidade de Software, Curitba, Brasil.

Nasir, M. H. N. Md., Ahmad, R. e Hassan, N. H. (2008) "Resistance in the Implementation of Software Process Improvement Project in Malaysia", Journal of Computer Science, vol. 4, issue 3, p.211.

Niazi, M., Wilson, D., Zowghi, D. (2006) "Critical success factors for software process improvement implementation: An empirical study", Software Process Improvement and Practice, v. 11, n. 2, pp. 193-211.

Robbins, S. P. (2005) “Comportamento organizacional”. 11. ed. São Paulo: Pearson Prentice Hall.

Seaman, C.B. (1999) "Qualitative methods in empirical studies of software engineering”, IEEE Transactions on Software Engineering, v. 25, n. 4, pp. 557-572.

Sebrae (2014) "Critérios de classificação de empresas". Disponível em: $<$ http://www.sebrae-sc.com.br/leis/default.asp?vcdtexto=4154>. Acesso em 20 abr. 2014.

Softex (2014) “Qualidade - MPS.BR”. Disponível em: <http://www.softex.br/mpsbr/>. Acesso em 18 abr. 2014.

Softex (2012) "MPS.BR - Guia Geral MPS de Software:2012". Disponível em: $<\mathrm{http}: / /$ www.softex.br/mpsbr/guias/>. Acesso em 18 fev. 2014.

Sommerville, I. (2011) “Software Engineering”. 9. ed. São Paulo: Pearson.

Viana, D., Júnior, D. C. V., Souza, C. de e Conte, T. (2011) "Aspectos humanos que afetam um programa de melhoria de processo de software: uma análise qualitativa", In: XIV Ibero-American Conference on Software Engineering, Rio de Janeiro, Brasil. 